\title{
Dementia with Lewy Bodies
}

National Institute of Neurological Disorders and Stroke (NINDS)

\section{Source}

National Institute of Neurological Disorders and Stroke (NINDS). Dementia With Lewy

Bodies Information Page.

Dementia with Lewy bodies (DLB) is one of the most common types of progressive dementia. The central features of DLB include progressive cog nitive decline,

"fluctuations" in alertness and attention, visual hallucinations, and parkinsonian motor symptoms, such as slowness of movement, difficulty walking, or rigidity. People may also suffer from depression. The symptoms of DLB are caused by the build-up of Lewy bodies - accumulated bits of alpha-synuclein protein -- inside the nuclei of neurons in areas of the brain that control particular aspects of memory and motor control. Researchers don't know exactly why alpha-synuclein accumulates into Lewy bodies or how Lewy bodies cause the symptoms of DLB, but they do know that alphasynuclein accumulation is also linked to Parkinson's disease, multiple system atrophy, and several other disorders, which are referred to as the "synucleinopathies." The similarity of symptoms between DLB and Parkinson's disease, and between DLB and Alzheimer's disease, can often make it difficult for a doctor to make a definitive diagnosis. In addition, Lewy bodies are often also found in the brains of people with Parkinson's and Alzheimer's diseases. These findings suggest that either DLB is related to these other causes of dementia or that an individual can have both diseases at the same time. DLB usually occurs sporadically, in people with no known family history of the disease. However, rare familial cases have occasionally been reported. 